\title{
BIO-EFFECTS OF AIRCRAFT NOISE AND ANTIOXIDANT ACTIVITY OF OMEGA3 ON MICE BRAIN
}

\author{
Mervat A. Mohamed \\ Biomedical Physics Department, Medical Research Institute, Alexandria University, \\ Egypt \\ Corresponding author's name: Mervat A. Mohamed
}

\begin{abstract}
In order to investigate the non-auditory bio-effects of aircraft noise exposure on brain, mice in soundproof chambers were exposed to a previously recorded aircraft-related noise signal for a duration of 1,2 or 3 weeks. For comparison, unexposed control mice have been used. The work plan includes aircraft takeoff and landing noise which adjusted to a level of $100 \mathrm{~dB}$ forthe 4 experimental groups for 2 hours daily. The recorded noise was analyzed using Audacity software program. The levels of blood glucose and cortisol in serum were measured. Morphological changes of brain tissue were observed by light microscope. The results showed that, Takeoff frequency ranged from 3-4 KHz at 100dB, while dominant frequency for landing was 2-3 KHz. Noise of $100 \mathrm{~dB}$ was found to cause significant increases in plasma glucose and cortisol for exposed mice as compared to no noise exposure. Administration of omega 3 for three weeks causes a significant improvement in plasma glucose and cortisol. Histopathological changes in brain of mice exposed to aircraft noise revealed some vesicles and mild to moderate apoptosis and pyknosis of the nuclei. In conclusion the present study determined that high intensity noise has a direct and clear effect on physical, biochemical values and thus noise exposure should be well organized.
\end{abstract}

\section{KEY WORDS:}

Aircraft noise, Stress, Omega 3, Bloodglucose, Cortisol, Histopathological changes.

\section{INTRODUCTION}

Noise is an unavoidable part of our daily lives and has increasingly become a major burden on the quality of lives. Noise, has been shown to produce a number of physiological, biochemical, and neurochemical rejoinder in both human and animals. High levels of noise can be associated with impairment of ability to concentrate [1, 2]. Aircraft noise has been shown to influence cardiovascular health in adults, and there is some evidence that the average blood pressure was significantly higher in the group with higher noise exposure [3]. Both long-term as well as heavy studies of animals have provided biological mechanism and plausibility for the theory that exposure to environmental noise affect the central nervous system and causes manifest diseases. The brain, the key organ that interprets and be interrogated to potential stressors, recognizes the sound levels and differentiate the stress levels. It reacts within split seconds timing to instruct the rest of the body in how to adjust to this stressful situation by the release of cascade of hormones [4,5].Investigation the physiological effects of aircraft noise under the condition of laboratory, indicated that medium and short term noise exposure $(<15$ day $)$ could produce anxiety on rats, however, long term noise exposure $(>30 \mathrm{~d})$ could produce depression or com or bid anxiety and

DOI: $10.5121 /$ ijbes.2016.3302 
depression on rats. However, the results of neurotransmitters showed that, Norepinephrine, NE and 5-hydroxytryptamine, 5-HT concentration of experimental group increased at the beginning, then decreased with the time [6].Furthermore, noise exposure for 15 days was reported to affectserotonin and dopamine levels in the hippocampus and produced recognition memory failure in rats[2].

The alteration in the levels of plasma corticosterone after different durations of noise exposure have been studied by Samson et al [5] to analyze their role in combating time -dependent stress effects of noise. Broadband white noise of $(100 \mathrm{~dB})$ exposure to male albino rats significantly increased the levels of plasmacorticoster one after different duration (acute, $1 \mathrm{~d}$; sub-acute, $15 \mathrm{~d}$; chronic, $30 \mathrm{~d}$ ) of noise exposure. Also, association between noise exposure and plasmacorticoster one levels was studied by Vitaleet al [7]. A 10 minutes exposure to noise stress resulted in increased corticosterone levels in mice plasma.

Observing the morphological changes in neuronal cells can assess the effects of noise. The detrimental effects of noise stress on both deterioration of epithelial cells and apoptosis of stromal cells in the brain have been well documented in several studies[7,8]. The findings of these studies indicated that aircraft noise exposure leads to the neurons of temporal lobe began to apoptosis, synapse morphology had changed, synaptic cleft of hippocampus and the temporal lobe area was vague, chromatin aggregated and a synaptic vesicle was not clear [7].Di et al[9] exposed rats to previously recorded aircraft-related noise at 75 and $80 \mathrm{~dB}$ for $65 \mathrm{~d}$. The results showed that the neuron and synaptic structures of the temporal lobe have signs of damage after aircraft noise of $80 \mathrm{~dB}$ exposure for 65 days.

Due to the high demand for energy from the brain, it would be susceptible to oxidation. Also neurons are sensitive to oxidative stress, as it is considered responsible for the occurrence of many neurological diseases and cell death [10].Omega-3 polyunsaturated fatty acids block apoptotic neural cell death. Therefore, they are considered a strong protective against deterioration of acute and chronic neurodegenerative. They reduce pro-inflammatory mediators, increase the production of anti-inflammatory mediators and reduce susceptibility to lipid peroxidation and oxidative stress in neural cells[11].

None of the aforementioned studies studied physical analysis of aircraft noise. In this study, actual aircraft noise was played back to laboratory mice. Analysis the frequency of the noise, blood glucose level, cortisol level in serum and cell morphology of the brain tissue with and without omega -3 were studied.

\section{Materials and Methods}

\subsection{Materials}

The work plan included, recording takeoffs and landings of aircraft from the following link www.soundjay.com as a source of noise and then it was run through loudspeaker inside anechoic chamber.

\section{Anechoic chamber:}

Figure 1 illustrated schematic diagram of anechoic chamber in which the mice were placed to be exposed to the noise. Homemade $70 \times 70 \times 70 \mathrm{~cm} 3$ plywood box as anechoic chamber was made, lined with polyurethane foam from all sides. The chamber contains illumination and ventilation system.15 W RMS loud speaker was installed on one side of the anechoic chamber, driven by the power amplifier (Model FUS- 432 RFL (Made in China) that can transmit the recorded specified 
noise with the required $\mathrm{dB}$ value (Fig 1). Noise level was measured by a sound level meter (Lutron electronic enterprise Co, LTD.) Model SL- 4010, 35to $130 \mathrm{~dB}, 3$ ranges, Data hold and an instrument which responds to sound in approximately the same way as the human ear, where it givesrepeatable measurement of sound level. The recorded noise was analyzed using Audacity software program (version 2.0.5) http// audacity.sourceforge.net. Repeating area of the sound waves with the full characterization was obtained though the software analysis.

\subsection{Animals}

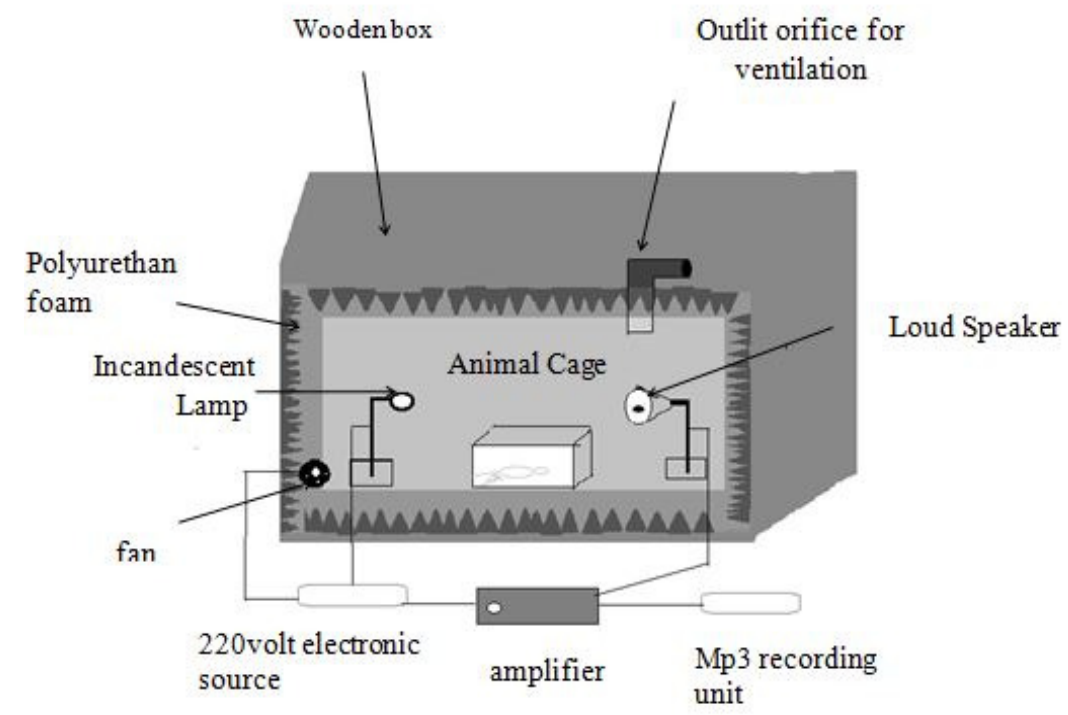

Use of experimental animals in this study was carried out in accordance with the ethical guidelines of the Medical Research Institute, Alexandria University, (Appendix2 Guiding principles for Biomedical Research Involving Animals, 2011).

\section{Animal Study Groups:}

Eighty mice weighted 25-30 gmwas purchased from the Experimental Animal Center of Medical Research Institute. The mice were kept in comfortable cages, well air conditioned, light system of $12 \mathrm{hr}$ day/ $12 \mathrm{hr}$ dark, food and water were allowed freely. Mice weredivided into three main groups. The division process was completely at random.

1-Control group was divided into two subgroups (10 mice each) as follow:

- Control group (A1) was remaining in the anechoic chamber for two hours daily without exposure to any sound.

- Control group (A2) was administered oral supplementation of daily dose of omega-3 fatty acid $100 \mathrm{mg} / \mathrm{kg}$ body weight, then remaining in the anechoic chamber for two hours daily without exposure to any sound.

2-Experimental group (B) consists of 30 mice was divided into three subgroups (10 mice each).

These three subgroups exposed to aircraft noise (takeoff and landing) at intensity of 100dB for 2 hours daily as follows: 
- GB1 for one week.

- GB2 for two weeks.

- GB3 for three weeks.

3-Experimental group (C) consists of 30 mice was divided into three subgroups (10 mice each).

These three subgroups administered oral supplementation of daily dose of omega- 3 fatty acid 100 $\mathrm{mg} / \mathrm{kg}$ body weight, then exposed to aircraft intensity of $100 \mathrm{~dB}$ as follows:

- GC1for one week.

- GC2 for two weeks.

- GC3 for three weeks.

\subsection{Biochemical Measurements:}

\subsection{1 - Glucose level measurement}

The glucose assay uses the glucose oxidase- peroxide reaction for determination of glucose concentrations by generation of a pink dye with an optimal absorption at 514nm [12]. Blood samples were taken from control and experimental groups during dissection. For plasma glucose analysis, the blood samples were collected in the gray tubes which contain sodium fluoride and potassium oxalate then kept in refrigerator at $-20{ }^{\circ} \mathrm{C}$ until analysis.

\subsection{2- Cortisol level measurement}

Due to the circadian rhythm of cortisol levels in serum, the sample collected in the morning. The trunk blood was centrifugedat $400 \mathrm{rpm}$ for 30 minutes at $4 \mathrm{oC}$, serum collected using standard sampling tubes, and stored at $-8^{\circ} \mathrm{C}$ until assayed. Before the determination of corticosterone (CORT), serum was diluted 3:1 in assay buffer and assayed in duplicate using commercially available Elecsys2010 (Sarstedt, Numbrecht, Germany).

\section{4- Histopathological studies:}

A cryosectioning of brain at low temperature $-60 \mathrm{oC}$, $(10 \mu \mathrm{m})$ thick using SLEE MAINZ; type MEV) cryostat with a stainless steel knife was used. The section was fixed at $10 \%$ formaldehyde then washed in dis.H2o. The nuclear fast red was used as a counter stain, then the slide left to dry and mounted by glycerine-gel and covered by cover slip to be observed under light microscope (BX41; Olympus America Inc). A photograph with digital compact camera (CAMEDIA C -7070, U- CMAD3, Olympus Company with high performance 7.1 megapixel colored camera was taken.

\section{3-Results}

\section{1-Analysis of aircraft noise:}

\subsection{1 -Band selection and expansion of takeoff and landing noise}

Figure 2 (a,b,c\&d) shows amplitude- time plots in both , time - unexpanded and time- expanded modes respectively. Amplitude is measured in arbitrary units, and the time in sec. The unexpanded plot contains screenshot of the entire recorded takeoff signal sound, with a vertical red line showing where the time-expanded sections were selected. The expanded waveform shows random variation in amplitude. 
International Journal of Biomedical Engineering and Science (IJBES), Vol. 3, No. 3, July 2016

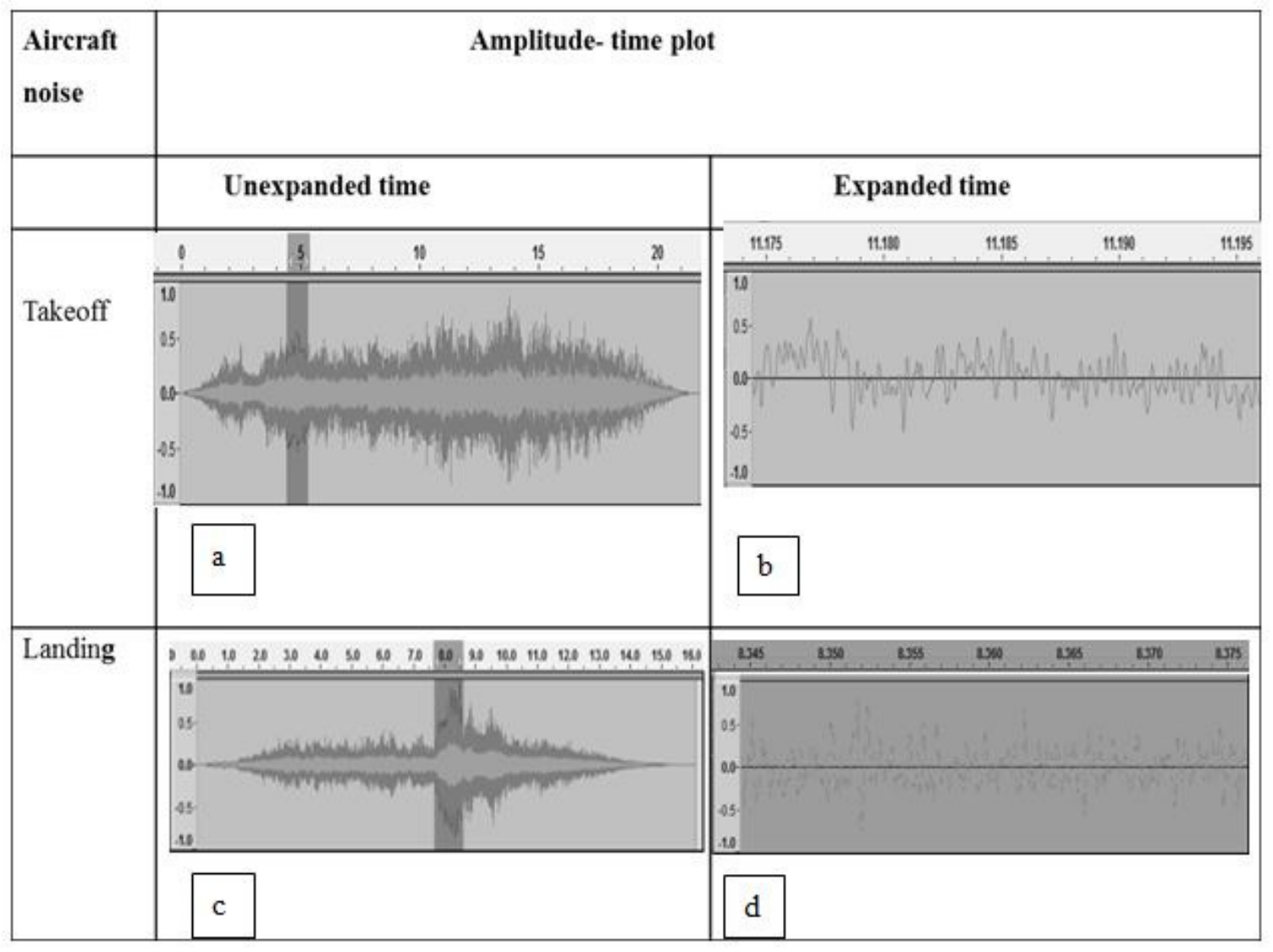

Figure.2.(a,b, c \&d): Take off noise amplitude-time plot in unexpanded and expanded mode

\subsubsection{Spectrum of takeoff and landing:}

A sound spectrum displays the different frequencies present in a sound.It is represent a short sample of a sound -in terms of the amount of vibration at each individual frequency. Figure 3 (a\&b) shows spectrum analysis of takeoff and landing noise. The $\mathrm{X}$ - axis represents the sound frequency in $\mathrm{Hz}$, while the $\mathrm{Y}$-axis represents the sound intensity in $\mathrm{dB}$. As shown from the figure the highest frequency ranged from 3to $4 \mathrm{KHz}$ and 2 to $3 \mathrm{KHz}$ for takeoff and landing respectively. 


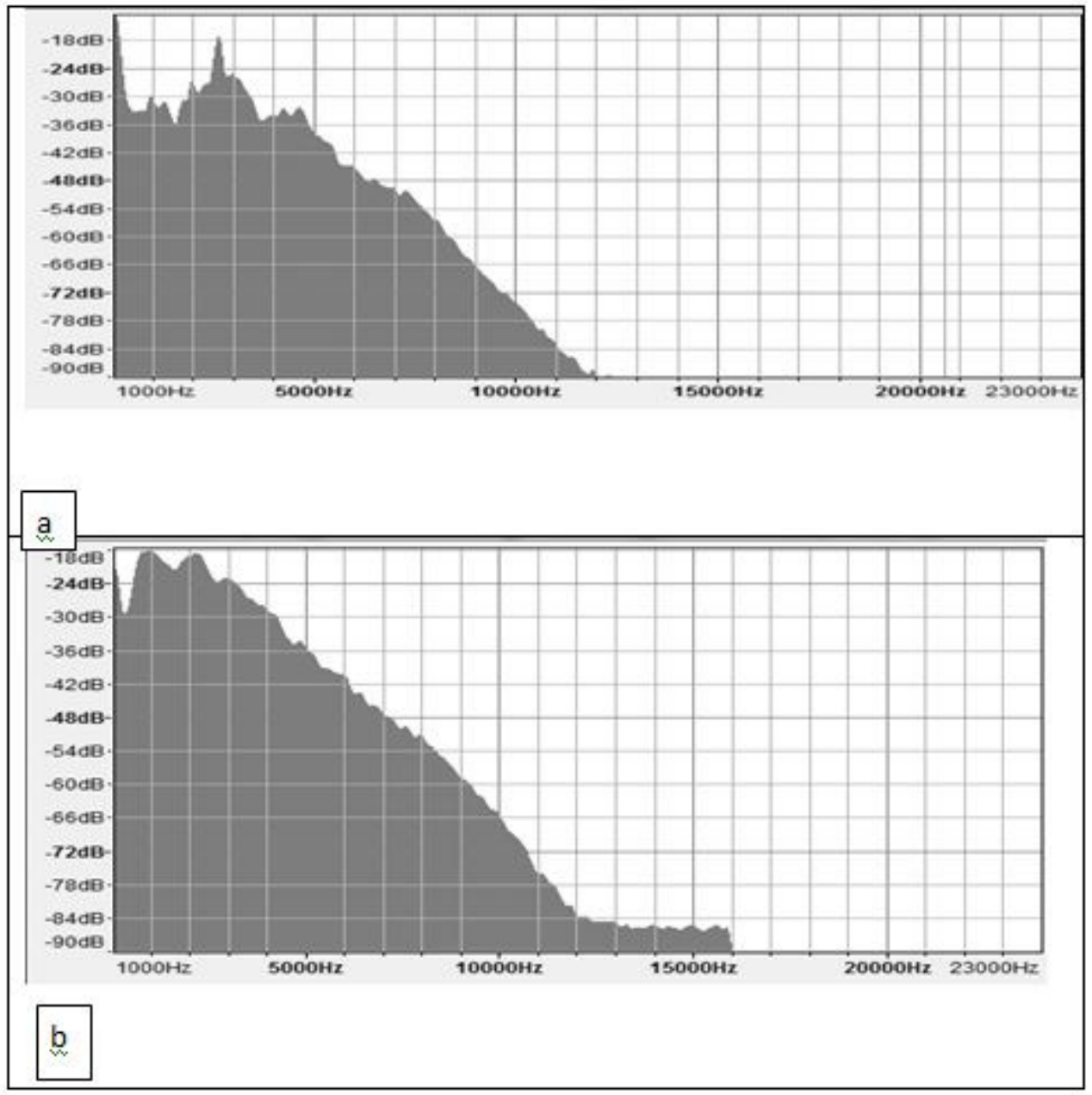

Figure $3(\mathrm{a} \& \mathrm{~b})$ :Spectrum analysis for takeoff and landing of aircraft noise

\subsection{3-Cepstrum analysis and standard Auto correlation:}

Cepstrum is an analysis function with many application in signal processing. It is used as a complementary tool to spectral analysis, it helps identifying items which not readily identified by spectral analysis. Takeoff and landing cepstrum analysis are shown in figure (4a\&c) for takeoff and landing noise. The cepstrum diagram shows sporadic very high spikes of sound that are closed to aircraft and or workers speech and any movement are separated to the left of the diagram as a characteristic band, while the constant annoying sound appears at the rest of the axis. As it is already known that autocorrelation measures, to what extent the sound repeats itself. In other words how many identical frequencies in the used sound sample. From the autocorrelation figure ( $4 \mathrm{~b} \& \mathrm{~d}$ ) of the selected takeoff and landing noise band, the higher level sound intensity is separated at the left side of the plot (unrepeated), while the continuous sound repeated in the rest of distinct bands. 


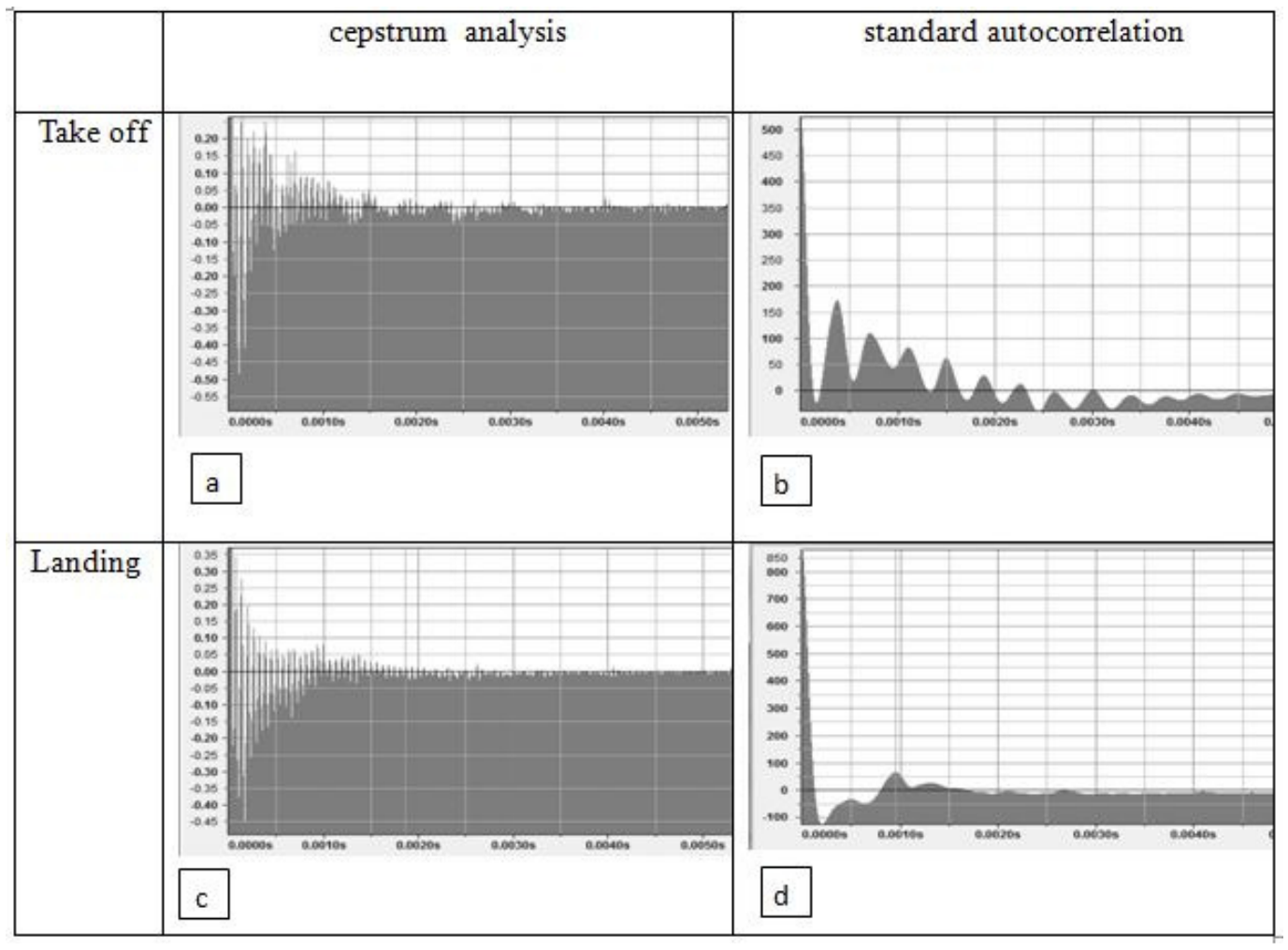

Figure 4.(a,b,c \& d) : Takeoff and landing cepstrum analysis and standard autocorrelation

\section{2- Biochemical studies:}

\subsection{1- Blood glucose level}

Blood glucose level $(\mathrm{mg} / \mathrm{dl})$ in all groups exposed to takeoff and landing noise with or without omega 3 were represented in Figure 5. It is clear that blood glucose, were significantly increased in its concentration for groups exposed to noise as compared to control $(\mathrm{p}<0.05)$. Groups that were administered omega- 3 before exposure to the sound did not give any significant reduction in the blood glucose level with the exception of group administered omega 3 for three weeks then exposed to takeoff and landing sound. Blood glucose of this group significantly decreased from $(212.4 \pm 22.8 \mathrm{mg} / \mathrm{dl}$ to $122.4 \pm 15.9 \mathrm{mg} / \mathrm{dl})(\mathrm{p}<0.05)$ as compared to groupexposed to takeoff and landing noise without omega.

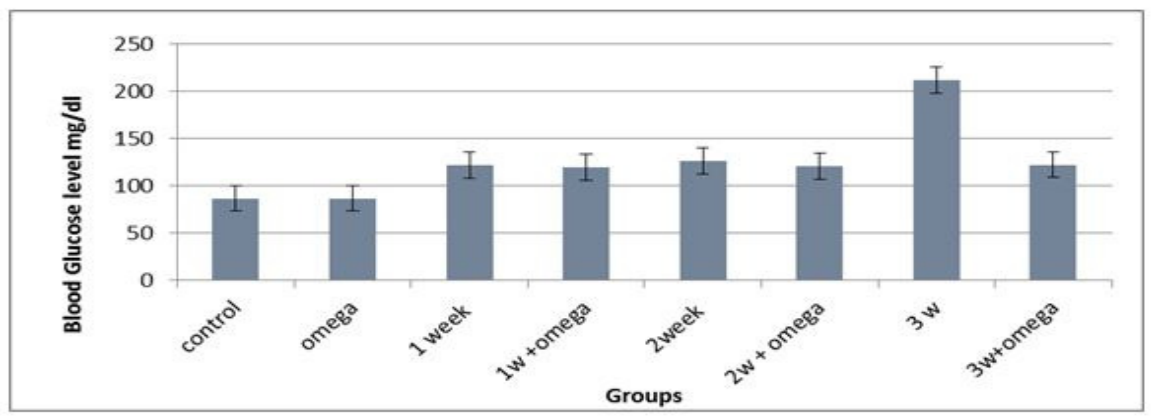

Figure 5: Effect of exposure to aircraft noise on blood glucose level 


\subsection{2- Cortisol level}

Figure 6 shows the relationship between the exposure of control and experimental groups to of aircraft noise at $100 \mathrm{~dB}$ intensity with different durations and the average concentration of plasma cortisol level. Data explained that there was significant difference in cortisol level with different groups when compared to control group $(\mathrm{p}<0.05)$. Groups administered omega 3 show no any significant decrease in cortisol level even after 3 weeks. Figure 7 shows linear relation between cortisol and blood glucose level, from this relation increase in blood glucose could be calculated from equation $\mathrm{Y}=38.7 \mathrm{x}-72.7$ where $\mathrm{y}$ is blood glucose and $\mathrm{x}$ is cortisol level with a correlation coefficient of $\mathrm{r}=0.8098$.

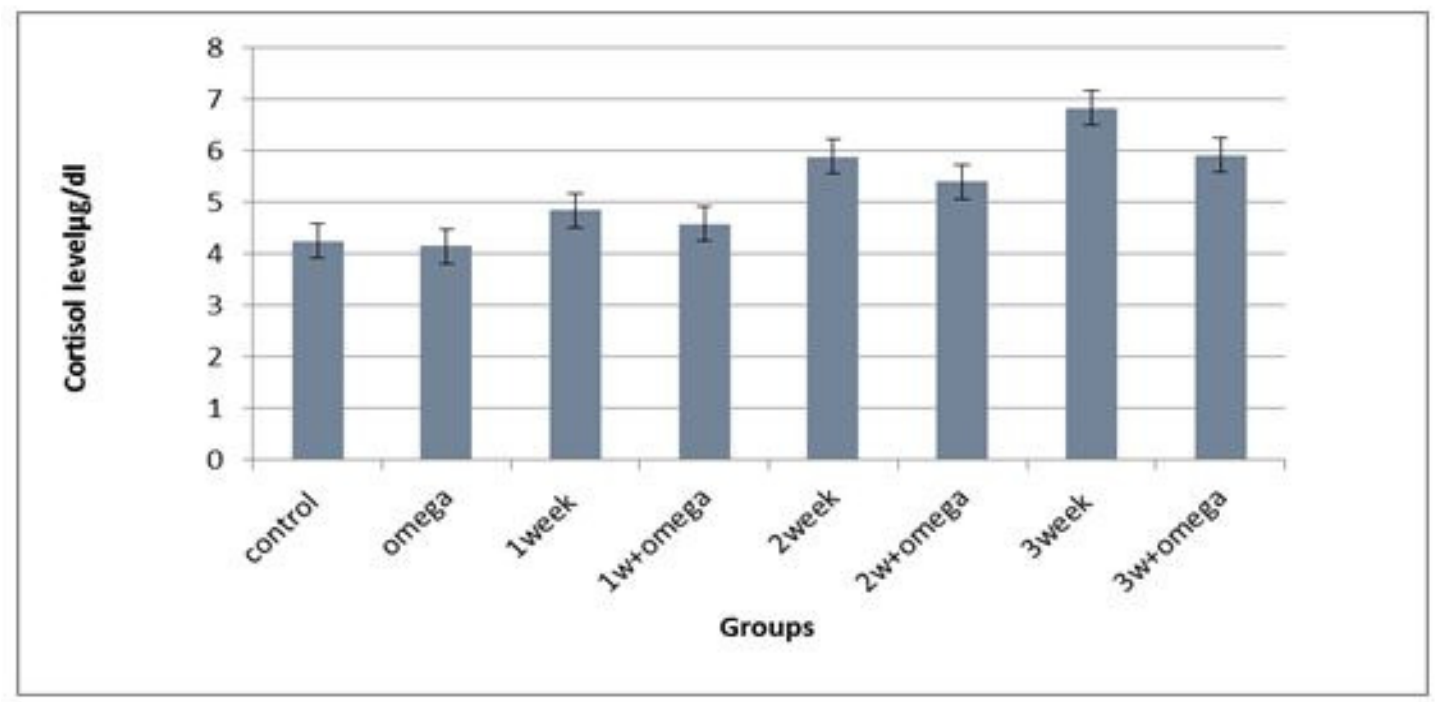

Figure. 6: Relationship between cortisol level and noise exposure duration in different group

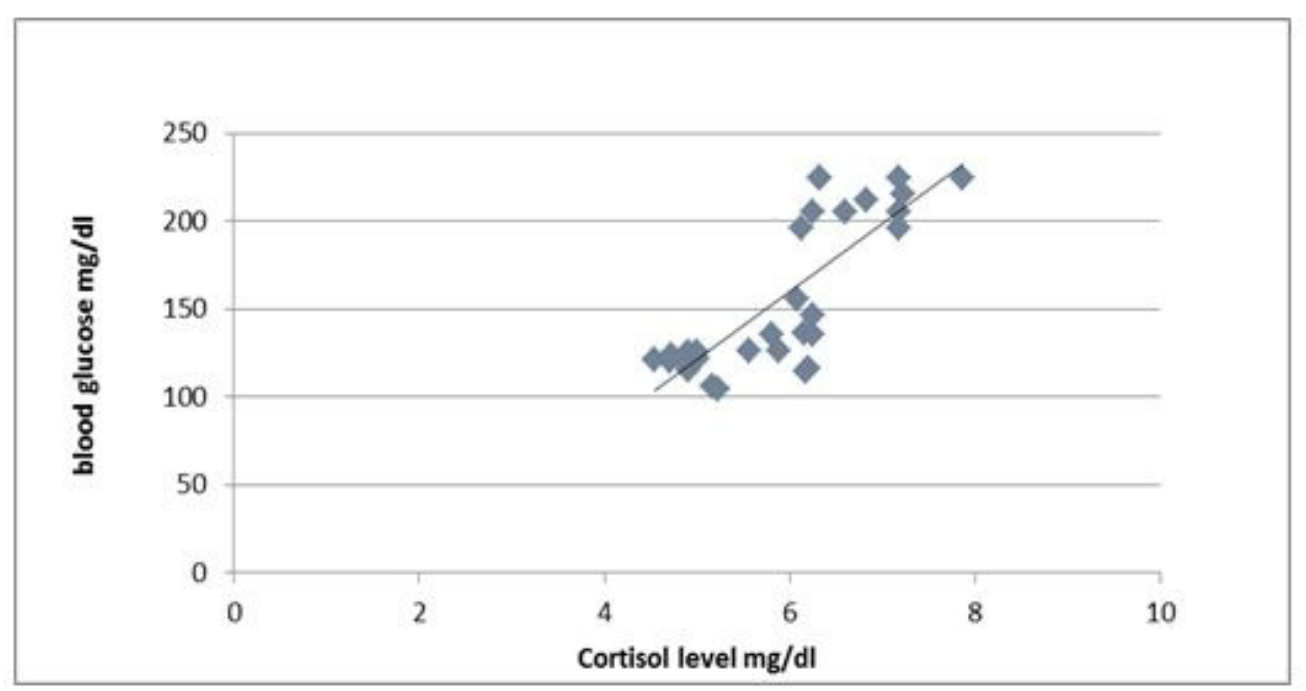

Figure 7: Linear relation between cortisol and blood glucose 


\subsection{3-Histopathological study}

The histopathological changes in brain of mice exposed to different duration one, two and three weeks with or without omega 3 to aircraft noise in comparison with control group were shown in figures (8-11). Light micrograph showed that mild to moderate vesicles, neurons of experimental group exposed to noise for three weeks began to pyknosis and apoptosis.

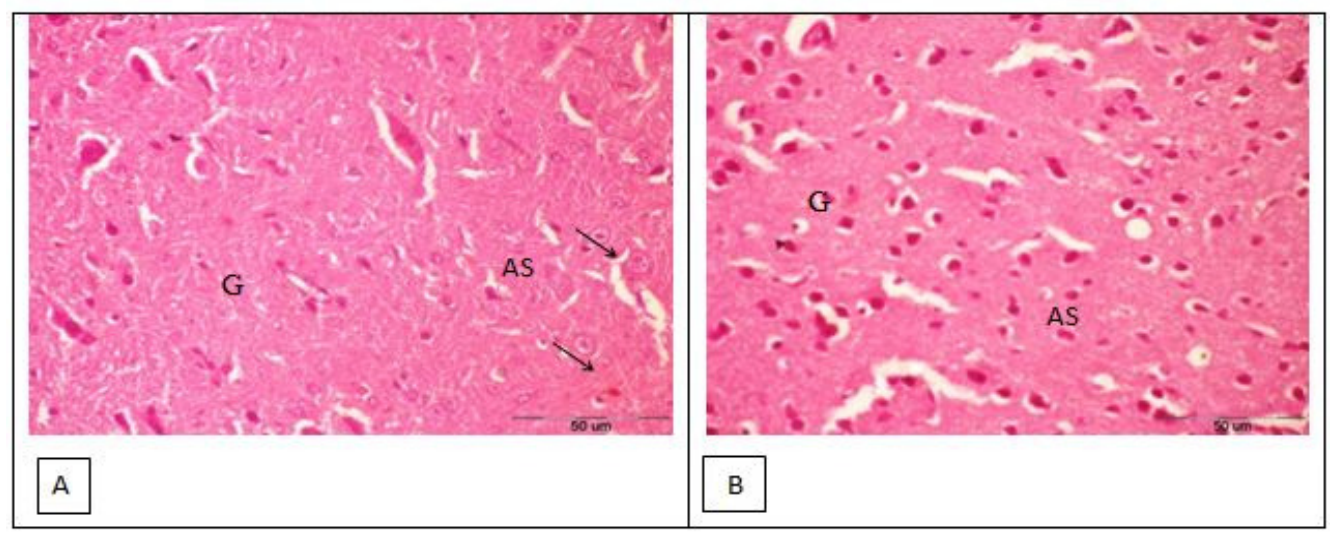

Figure (8) photomicrograph of control group reveals normal brain tissue formed of glial tissue (G) and astrocytes (arrows AS). A: Normal brain, B:Normal brain with omega 3.

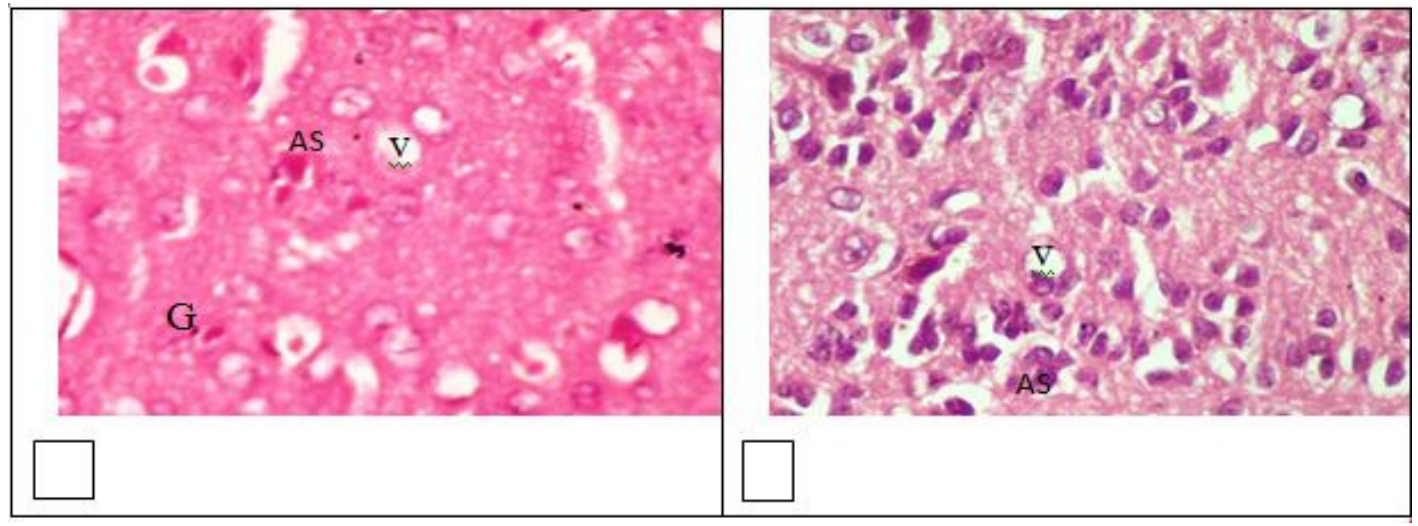

Figure 9: Photomicrograph of group exposed to noise for one week.A: without omega, B: with omega) reveals normal glial tissue $(\mathrm{G})$ and astrocytes (AS) with some vesicles (V).

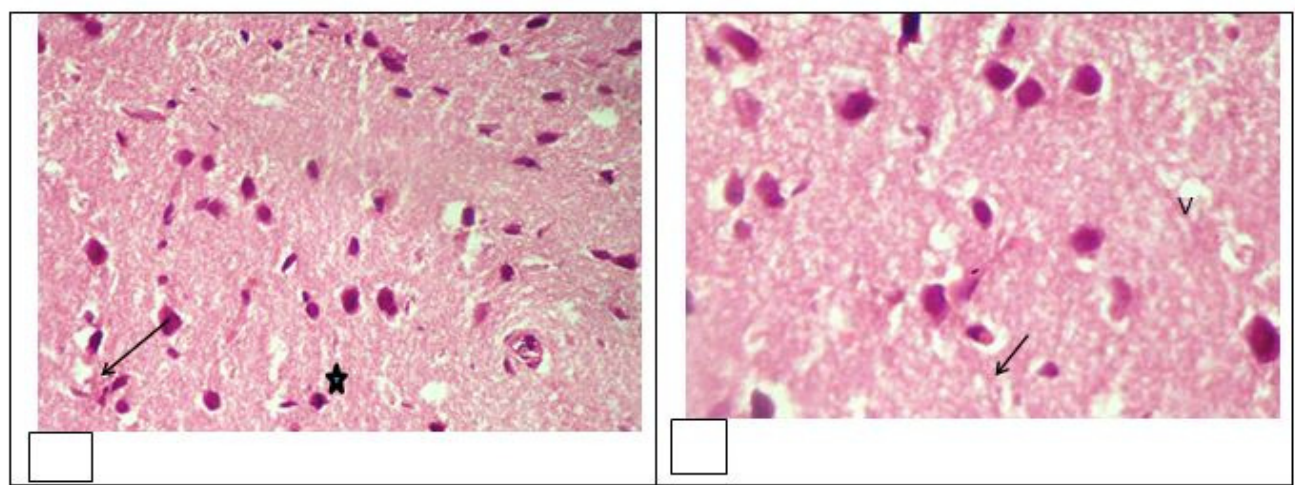

Figure 10: Photomicrograph of group exposed to noise for two weeks. A: without omega, B: with omega) reveals apoptotic nuclei (arrow) and pyknoticcell $(*)$ with many vesicles (v). 


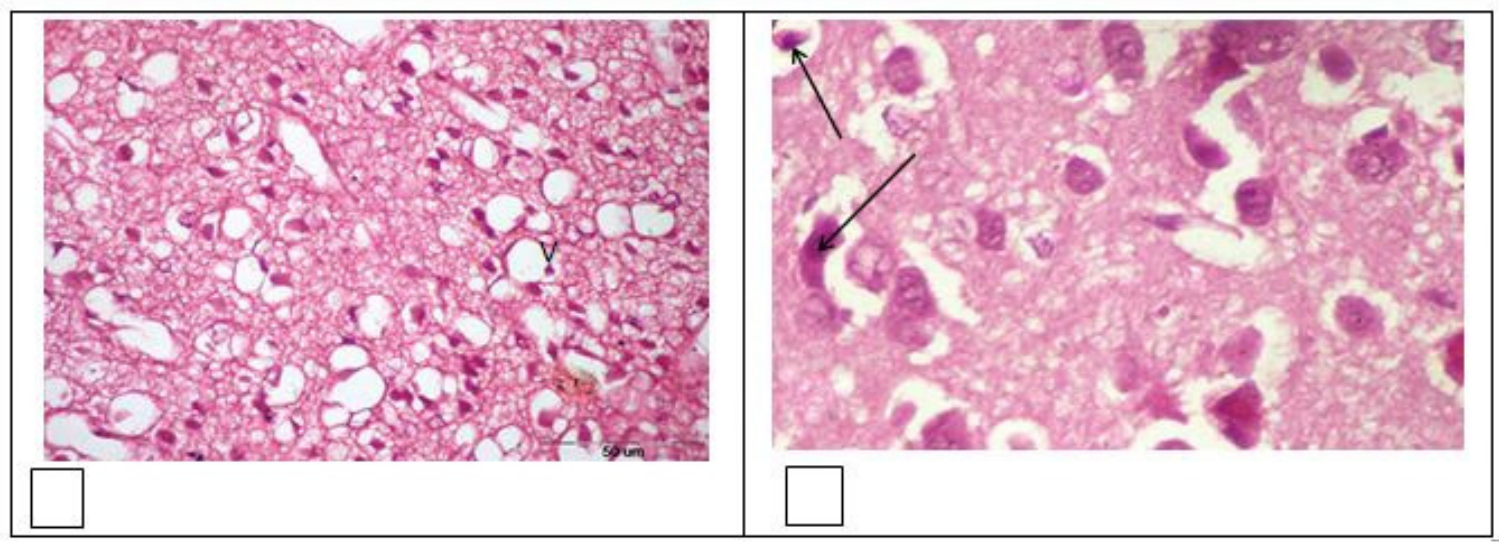

Figure 11:Photomicrograph of group exposed to noise for three weeks.A:without omega, B: with omega) showing fragmentation of nuclear membrane with pyknosis of nuclei (arrow) and with numerous vesicles (V).

\section{DISCUSSION}

Occupational noise is one of the main annoying stressor to which a large population is being exposed for a long duration daily. Many studies have reported that exposure to aircraft noise causes many health problems for populations working in or near airport such as cardiovascular diseases, lack of concentration, fatigue, heart trouble, plasma viscosity, high glucose level and reduced motor efficiency [13].

Noise usually consists of sound signals with a broad spectrum, including both low frequency and high frequency region [14].A disreputable example of low frequency noise is the noise generated bythe take-off rollof aircraft on the runway,this type of aircraft noise known as aircraft groundnoise[15].

The present study was undertaken to analyze noise signals and spectrum of takeoff and landing of aircraft. According tofigure 2 (a,b,c,and d) shape of aircraft noise has a repeated signals with different intensities and different frequencies. The spectrum of noise emitted from takeoff has the highest levels in the frequency band ranged from $3 \mathrm{KHz}-4 \mathrm{KHz}$ at $100 \mathrm{~dB}$ and gradually decrease above this frequency. The spectrum of noise emitted from landing has the highest levels in the frequency band ranged from $2-3 \mathrm{KHz}$ at $100 \mathrm{~dB}$ and fall off more sharply above this frequency at 4KHzto 83.58dB.This analysis is a good agreement with Berglund et al [16] study which revealed that, although the spectra are often not linear, aircraft groundnoise spectra measured in communities near an airport are nearly linear at a frequency ranged from 1-2 kHz andthen fall off more steeply above this frequency.Starting with the effect of noise on blood glucose level, noise exposure is a stressful condition and stress is known to signal the body to raise blood glucose level in order to generate energy to respond to the stress [17]. The relationship between the effect of noise and its impact on blood glucose has been studied. The results showed that,an elevation of the plasma blood glucose for all groups' noise exposure as compared to control. The study also showed that the three weeks noise exposure group had a highest level of plasma blood glucose than that of control, one week and two weeks with or without omega 3 supplement as shown in figure (5). Blood glucose level also increased in the study held by Emily and Mark[18]theirresults revealed that, there was an increase in blood glucose level after exposure toan aircraft noise of a maximum level $105 \mathrm{~dB}$ for 3 seconds.Building upon Muayad et al [19] 
studies, the effects of acute and chronic loud noise exposure ranged from 80-100 dBwas found to cause high levels in plasma blood glucose as compared to no noise exposure.

Cortisol plays a vital role inhealth maintenance. But in order to do so, circulating levels must be maintaining levelswithin a narrow range. If levels less than optimal, signs and symptoms of adrenal weariness occur; if levels increased and remain above optimal for a period of time, signs and symptoms of metabolic syndrome occur [20] .

Raising in cortisol levels have been found in humans and animals that exposed to aircraft noise or road traffic noise [21].The present study observed the effect of noise on cortisol as stress hormone, where there was an elevation of cortisol for all groups' noise exposure as compared to control. The study also showed that the three weeks noise exposure group had a highly level of cortisol in comparison to control, one week and two weeks with or without omega 3 supplement as shown in figure (6). The aforementioned results are similar to those reported byHartono[22] experiment where group exposed to aircraft noise at an intensity level of $92.29 \mathrm{~dB}$ the noise acted as a stressor, resulting in a higher increase in mean cortisol level than was the case with aircraft noise at an intensity level of $71.49 \mathrm{~dB}$. Furthermore, Cheng and Ariizumi[23] who did an experiment with BALB/c mice at noise intensity level of $90 \mathrm{~dB}$, with a length of exposure of five hours per day for four weeks. Mean of cortisol level of treated group exposed to noise was 4.25 $\mu \mathrm{g} / \mathrm{dl}$, which was higher than that of the control group of $1.60 \mu \mathrm{g} / \mathrm{dl}$.

There is a strong relationship between cortisol and blood glucose levelsas shown in figure (7). Under stressful condition, sufficient concentrations of cortisol mobilize glucose into the bloodstream via gluconeogenesis, reduced insulinsensitivity, amino acid motivation and protein catabolism. Cortisol obtains quick glucose for the body to use in times of stress. Also, in stress time cortisol gets rapid glucose for the body to use while reduce insulin effects, so blood glucose levels becomes high and insulin unable to perform its regular function of maintaining normal glucose levels[24].On the other hand, according to this relationship, diet plays a main role in adrenal health. Poor eating habits and food choices not only leave the body without essential nutrients but also impede or create a burden on the stress response, digestive, energy production and other homeostatic systems. There are specific dietary guidelines that form the other side of the therapeutic effect [25]. In the present study omega 3 was used as diet supplements, the results indicate that blood glucose and cortisol decreased after intake of omega for three weeks as shown in figure $(5,6)$. This study is a good agreement with Delarue, et al [26]study, which evaluated the effect of n-3 fatty acids on the hypothalamic-pituitary-adrenal (HPA)axis. Their results indicated supplementation with $7.2 \mathrm{~g}$ of fish oil per day, the cortisol response to stress was significantly reduced after 3 weeks.

\section{CONCLUSION}

Depending on the present study, aircraft noise may lead to the negative effect on blood glucose level and cortisol level, particularly in long term noise exposure. Also, there was a linear relationship between circulating cortisol levels with blood glucose level due to depletion of insulin resistivity. Moreover, the study indicated that aircraft noise exposure leads to histological changes in neuronal structures of the mice brain. Increased natural antioxidantand neuro protective like Ginkgo biloba or Panax ginseng intake to reduce the oxidant stress produced by noise. It must be taken in consideration, increased improvements and control methods from noise based on intensity and frequency levels. 
International Journal of Biomedical Engineering and Science (IJBES), Vol. 3, No. 3, July 2016

\section{REFERENCES}

1. Ravindran R, Rathinasamy SD, Samson J, Senthilvelan M.,( 2005).Noise stress induced brain neurotransmitter changes and the effect of osmium sanctum (Linn) treatment in albino rats. J Pharmacol Sci.; 98, 354-360.

2. Haider S, Naqvi F, Batool Z, Tabassum S, Perveen T, Saleem S, Darakhshan J. H.,(2012).Decreased Hippocampal 5-HT and DA Levels Following Sub-Chronic Exposure to Noise Stress: Impairment in both Spatial and Recognition Memory in Male Rats.Sci Pharm; 80, 1001-11.

3. Y. Aydin and M. Kaltenbach.,(2007).Noise perception, heart rate and blood pressure in relation to aircraft noise in the vicinity of the Frankfurt airport.Clin Res Cardiol;96, 347-358.

4. McEwen BS., (2000). The neurobiology of stress: from serendipity to clinical relevance. Brain Res.; 886, 172-189.

5. Samson J, Sheeladevi R, Ravindran R, Senthilvelan M.,(2007).Stress response in rat brain after different durations of noise exposure. Neurosci Res.; 57, 143-147.

6. L Jia., (2011).Effects of aircraft noise exposure on recipients in relation to behaviors, neurotransmitters and morphology of nerve tissues in brain. Master Thesis.

7. Vitale, G., Arletti, R., Sandrini, M., (2005). Acute noise stress analgesia in relation to 5-HT2 and $\mu$-opioid receptor changes in the frontal cortex of young mice. Life Sci.,77, 2500-2513. [doi:10.1016/j.1fs.2005.01.031]

8. Akdogan, O., Selcuk, A., Take, G., Erdoğan, D., Dere, H., (2009).Continuous or intermittent noise exposure, does it cause vestibular damage? an experimental study. AurisNasus Larynx, 36,2-6.

9. Guo-qing DI $\dagger$, Bing ZHOU, Zheng-guang LI, Qi-li LIN., (2011).Aircraft noise exposure affects rat behavior, plasma norepinephrine levels, and cell morphology of the temporal lobe. Journal of Zhejiang University-SCIENCE B (Biomedicine \& Biotechnology) 12,969-975.

10. Butterfield DA, Stadtman ER., (1997). Protein oxidation processes in aging brain. Adv Cell Aging Gerontol2, 161-91.

11. El-Ansary KA, Al-Daihan KS, El-Gezeery RA., (2011).On the protective effect of omega-3 against propionic acid-induced neurotoxicity in rat pups.Lipids in Health and Disease, 10: 142-52.

12. McMillin JM., (1990). Blood glucose. In: The History, Physical and Laboratory Examination. Walker HK, Hall JW (eds). 3rd Ed. Butterworths, Boston., 622-5.

13. Subroto S. Nandhi, Sarang V. Dhatrak.,(2008). Occupational noise-induced hearing loss in India. Indian J of Occup Environ Med, 12,53-56.

14. Buikema, E.; Vercammen, M.; van der Ploeg, F.; Granneman, J.; Vos, J. ,(2010). Development of a rating procedure for low frequency noise: results of measurements near runways. In Proceedings Internoise, Lisbon, Portugal, 13-16 June 2010; unpaginated, 9 pp. (on CD).

15. Welkers, D., (2010). Another Kind of Noise: Groundnoise. In Proceedings Internoise, Lisbon, Portugal, 13-16 June 2010; unpaginated, 6 pp. (on CD).

16. Berglund, B.; Hassmén, P.; Soames Job, R.F., (1996).Sources and effects of low-frequency noise.J. Acoust. Soc. Am.,99, 2985-3002.

17. Babisch, W. (2000). The noise/stress concept, risk assessment and research needs. Noise \& Health Journal, 4, 1-11.

18. Emily, G., \& Mark, J. (2006). Noise exposure, music, and animals in the Laboratory. A commentary based on laboratory animal refinement and enrichment forum (LAREF)discussions. Applied Animals Welfare Science Journal, 9, 327-332.

19. Muayad S. Rahma, Ni Ni Win, H. M, Rafidah and Razali, Ailin., (2011) The Effects of Noise on Biochemical Parameters using Rat`s Hearts. European Journal of Scientific Research,56, 93-96

20. Wilson JL.,(2001).Adrenal fatigue: the 21st century stress syndrome. Petaluma, CA: Smart Publications.

21. Spreng, M.,( 2000). Possible health effects of noise induced cortisol increase. Noise Health, 2,5963.

22. Hartono, M.Si.,(2010). Cortisol level decreases natural killer cell activity among women exposed to aircraft noise.Univ Med;29,153-61. 
International Journal of Biomedical Engineering and Science (IJBES), Vol. 3, No. 3, July 2016

23. Cheng Z, Ariizumi M.,(2007).Modulation of immune functions and oxidative status induced by noise stress. J Occup Health;49,32-8.

24. Weber-Hamann B, Kopf D, Lederbogen F., (2005).Activity of the hypothalamus-pituitary adrenal system and oral glucose tolerance in depressed patients.Neuroendocrinology;81,200-204.

25. James L. Wilson DC. (2014). Clinical perspective on stress, cortisol and adrenal fatigue Advances in Integrative Medicine, 1, 93-96.

26. Delarue J, Matzinger O, Binnert C, Schneiter P, Chioléro R, Tappy L., (2003). Fish oil prevents the adrenal activation elicited by mental stress in healthy men. Diabetes Metab.;29,289-295. 